\title{
Stability analysis of high power factor Vienna rectifier based on reduced order model in $d-q$ domain
}

\author{
Chaoliang DANG ${ }^{1}$, Xiangqian TONG ${ }^{1}$, Weizhang SONG ${ }^{1}$, Jingjing HUANG ${ }^{1}$
}

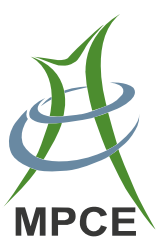

\begin{abstract}
For a DC distributed power system, system stability can be predicted by dividing it into source and load subsystems, and then applying the Nyquist criterion to the impedance interaction between the source and load model. However, the generalized Nyquist criterion is extremely complicated and cannot directly reveal effective control strategies to reduce interaction problems of cascade threephase AC systems. Specifically, as a current force rectifier, this characteristic makes it difficult to judge the stability of a cascade three-phase Vienna AC system. To deal with the aforementioned problems, a simplified small signal stability criterion is presented for an AC distributed power system. Based on the criterion, the small signal model and impedance based on the reduced order model in the $d$ $q$ domain are studied theoretically. For the instability issue, an impedance regulator design method is presented. The correctness of the simplified stability criterion and the effectiveness of the proposed impedance regulator method are validated by extensive simulation and experiment.
\end{abstract}

CrossCheck date: 16 August 2018

Received: 11 October 2017/Accepted: 16 August 2018/Published online: 30 October 2018

(C) The Author(s) 2018

$\triangle$ Chaoliang DANG

dangclkk@163.com

Xiangqian TONG

lstong@mail.xaut.edu.cn

Weizhang SONG

songwz464237@126.com

Jingjing HUANG

395367347@qq.com

1 Department of Electrical Engineering, Xi' an University of Technology, Xi'an 710048, China
Keywords Three-level Vienna rectifier, Small-signal stability, Nyquist criterion, Grid impedance, Impedance regulator

\section{Introduction}

In recent years, with the fast development of communications technology, the electric vehicle (EV) charging station and the computer industry, the safe and reliable operation of high voltage direct current (HVDC) power supply systems has become an important research topic all over the world [1, 2]. Specifically, the three-phase threelevel boost-type Vienna rectifier [3] has proven to be a cost-effective and very efficient solution, maximizing the power density of industrial motor drives, active filters, the EV charging station and data center HVDC power supply system [4]. However, power electronic converter systems with regulated output voltage feature negative incremental input impedance, which translates into a constant power load (CPL) behavior. This characteristic may makes the distributed power system (DPS) suffer from small-signal instability issues because of the dynamic interactions between the converters and passive components in the systems $[5,6]$. This issue exists in both DC and AC DPSs. For the DC DPS, the impedance criterion was first established by Middlebrook, who states that the system stability can be predicted by dividing it into the source and load subsystems, and then applying the Nyquist criterion to the ratio between the source output impedance and the load input impedance [7-9]. For the AC DPS, however, there has been much less research.

It should be noted that the definition of the small-signal impedance in three-phase AC DPS is complicated compared to the DC DPS. This makes it extremely complicated 
and cannot directly suggest effective control strategies to improve interaction problems of the source and load subsystems. Most of the work on the AC DPS can be categorized into two approaches. One is the analysis of the system in the $d-q$ domain, whereas the other applies harmonic linearization in the phase domain through symmetric components [10]. The impedance-based stability criteria developed by Macfarlane and Postlethwaite in the 1970's has also been proposed for the three-phase AC DPS. This method employs the generalized Nyquist criterion (GNC) in the $d-q$ domain to study the stability of a cascaded AC system [11]. However, calculating the singular value of the matrix was complicated and inconvenient for the design of AC DPSs. A significantly improved modeling method was presented by Mao in 1996 [12, 13]. By developing a reduced-order (RO) model for active front-end (AFE) converters, Mao showed that the GNC could be used to study the stability of the converter using the RO model [14-16]. In return, the stability of an AC DPS can be predicted by examining the locus described by the impedance models based on analytical calculations or numerical simulation. As a result, the RO model and impedance analytical calculations are critical for the design of threephase power conversion systems. With the rapid development of digital controllers, various digital control strategies have been proposed for the control of the Vienna rectifier [4, 17-19]. However, as a current force rectifier, detailed analysis on the RO averaged model and small signal stability analysis of the Vienna rectifier have rarely been reported.

In order to further simplify the theoretical analysis, this paper proposes a simplified stability criterion and an input impedance regulator design method to analyze the interaction problems between three-phase Vienna AC systems and the variable grid impedance. First, averaging and local linearization techniques are used to derive the dynamic RO model expressed in the $d-q$ domain. Then the impedance model is computed neglecting interactions between the $d$ $q$ components of control inputs and currents, respectively. For unstable cascade AC systems, an input impedance regulator design method is proposed to improve the interaction problem of the system. Most importantly, the derived small-signal stability criterion and impedance regulator provide a simple and useful theoretical basis for the design process. Instead of trial and error, the proposed criterion can be used to predict and guarantee the stability operation of an AC DPS during the design process, and though this paper is mainly concerned with the three-phase boost-type rectifier, the approaches can be applied to other topologies.

The contribution is organized as follows. First, a simplified small signal stability criterion for AC DPS is presented in Section 2. Then the RO small signal and impedance model are discussed, neglecting the interactions between the $d-q$ components, in Sections 3 and 4, respectively. The stability issue between the Vienna rectifier and grid impedance is proposed in Section 5. Then, for unstable systems, an input impedance regulator design method is discussed. Finally, the analysis of previous sections is verified in Section 6.

\section{Simplified small signal stability criterion for AC system in synchronous $d-q$ frame}

The AC DPS studied is shown in Fig. 1. The source subsystem is composed of a three-phase voltage source and LC filters, and the three-phase Vienna rectifier acts as a CPL and includes six diodes and three bidirectional switching units $Q_{\mathrm{a}}, Q_{\mathrm{b}}$, and $Q_{\mathrm{c}}$. Here, $u_{\mathrm{s} x}$ (subscript $x$ denotes $\mathrm{a}, \mathrm{b}, \mathrm{c}$ ) is the electromotive force of the power grid, $u_{\mathrm{g} x}$ is the grid voltage at the point of common coupling (PCC), $i_{\mathrm{g} x}$ is the grid current, $L$ is the inductance which is being used to suppress the high-frequency harmonics, $L_{\mathrm{sg}}$ denotes the equivalent inductance of the grid power, $C_{\mathrm{g}}$ denotes the distributed capacitance of the grid power, $R_{\mathrm{L}}$, $R_{\mathrm{sg}}$ and $R_{\mathrm{cg}}$ denote the parasitic resistances, $C_{1}$ and $C_{2}$ are the DC-link capacitors, $i_{\mathrm{op}}$ and $i_{\mathrm{on}}$ are the output currents, and $u_{0}$ is the output voltage of the DC bus.

The block diagram of the small signal model studied is shown in Fig. 2. $\boldsymbol{u}_{\mathrm{s} d q}(s)$ is the disturbance of the source subsystem. $\boldsymbol{Z}_{\mathrm{s} d q}(s)$ is the output impedance of the source subsystem seen from the PCC. $\boldsymbol{Y}_{\mathrm{L} d q}(s)$ is the input admittance of the Vienna rectifier seen from the PCC. $\boldsymbol{u}_{\mathrm{b} d q}(s)$ is the grid voltage at the PCC in the $d-q$ domain. $\boldsymbol{G}_{\mathrm{s} d q}(s)$ is the voltage gain of the input filter. $\boldsymbol{G}_{1 d q}(s)$ is the voltage gain of the Vienna rectifier.

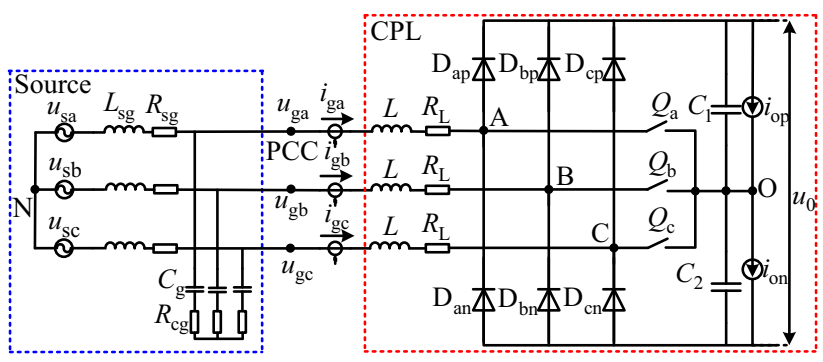

Fig. 1 Three-phase Vienna rectifier AC system

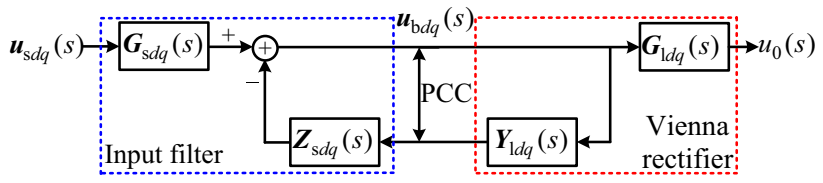

Fig. 2 AC DPS based on terminal characteristics of source subsystem and load subsystem 
The transfer function of the PCC voltage described in Fig. 2 can be written as:

$\boldsymbol{u}_{\mathrm{b} d q}(s)=\left(\boldsymbol{I}+\boldsymbol{Z}_{\mathrm{s} d q}(s)\left(\boldsymbol{Y}_{1 d q}(s)\right)^{-1}\right) \boldsymbol{G}_{\mathrm{s} d q}(s) \boldsymbol{u}_{\mathrm{s} d q}(s)$

Generally, the subsystems of source and CPL are stable. As the voltage transfer function matrix is designed to guarantee the stable operation of the system, there is no right-half plane pole on $\boldsymbol{Z}_{\mathrm{s} d q}(s)$ and $\boldsymbol{Y}_{\mathrm{L} d q}(s)$. As a result, the stability of the AC DPS is mainly described by the transfer matrix as:

$\varphi(s)=\boldsymbol{I}+\boldsymbol{Z}_{\mathrm{s} d q}(s)\left(\boldsymbol{Y}_{1 d q}(s)\right)^{-1}$

To ensure the stabilization of a feedback system, a negative real part must be included in the eigenvalues of (3) as:

$\operatorname{det}\left(\boldsymbol{I}+\boldsymbol{Z}_{\mathrm{s} d q}(s) \boldsymbol{Y}_{1 d q}(s)\right)=0$

Generally, the three-phase Vienna rectifier is a multiple input multiple output (MIMO) system, and the transfer matrix of a MIMO system is not a diagonal matrix. Then, for the AC DPS, its stability can be directly predicted by studying the return-ratio matrix defined as:

$$
\begin{aligned}
\boldsymbol{L}(s) & =\boldsymbol{Z}_{\mathrm{s} d q}(s) \boldsymbol{Y}_{1 d q}(s) \\
& =\left[\begin{array}{ll}
\boldsymbol{Z}_{\mathrm{s} d d}(s) & \boldsymbol{Z}_{\mathrm{s} d q}(s) \\
\boldsymbol{Z}_{\mathrm{s} q d}(s) & \boldsymbol{Z}_{\mathrm{s} q q}(s)
\end{array}\right]\left[\begin{array}{cc}
\boldsymbol{Y}_{1 d d}(s) & \boldsymbol{Y}_{1 d q}(s) \\
\boldsymbol{Y}_{1 q d}(s) & \boldsymbol{Y}_{1 q q}(s)
\end{array}\right]
\end{aligned}
$$

Since the three-phase Vienna rectifier is inherently a MIMO system, the GNC is usually very complicated because of the extreme complexity of the expressions of $\boldsymbol{Y}_{1 d q}(s)$ and $\boldsymbol{Z}_{\mathrm{s} d q}(s)$. Providing that the three-phase Vienna rectifier is fully decoupled by unity-power factor control, as compared with $\boldsymbol{Y}_{\mathrm{Lqq}}(s)$ or $\boldsymbol{Y}_{\mathrm{L} d d}(s)$, the value of the cross-coupled input admittances $\boldsymbol{Y}_{\mathrm{L} d q}(s)$ and $\boldsymbol{Y}_{\mathrm{L} d q}(s)$ can be ignored [20, 21]. By neglecting the cross-coupling between the input and output variables of the rectifier, the three-phase Vienna rectifier can be divided into two independent DC-DC channels. As a result, the small signal stability is derived as:

$\boldsymbol{L}(s)=\left[\begin{array}{ll}\boldsymbol{Z}_{\mathrm{s} d d}(s) & \boldsymbol{Z}_{\mathrm{s} d q}(s) \\ \boldsymbol{Z}_{\mathrm{s} q d}(s) & \boldsymbol{Z}_{\mathrm{s} q q}(s)\end{array}\right]\left[\begin{array}{cl}\boldsymbol{Y}_{1 d d}(s) & \mathbf{0} \\ \mathbf{0} & \boldsymbol{Y}_{1 q q}(s)\end{array}\right]$

Based on the assumption above, the three-phase Vienna rectifier can be independent of the $d-d$ and $q-q$ channel. As presented in $[9,13,20-22]$, since the phase of $\boldsymbol{Z}_{\mathrm{s} q q}(s) \boldsymbol{Y}_{1 q q}(s)$ was always leading the phase of $\boldsymbol{Z}_{\mathrm{s} d d}(s) \boldsymbol{Y}_{\mathrm{Ldd}}(s)$ by $180^{\circ}$, then the stability of the converter and filter system could be studied by analyzing $\boldsymbol{Z}_{\text {sdd }}(\mathrm{s}) \boldsymbol{Y}_{1 d d}(\mathrm{~s})$ alone. This validated the use of the RO model of the three-phase boost rectifier, which modeled the converter as an equivalent dc-dc converter. The stability of the converter and filter system can then be evaluated using the standard Nyquist stability theorem for a single input single output (SISO) system proposed in Middle-Brook's criterion for DC-DC converters. Moreover, modeling is of great importance since it is a first step toward control design, and the control scheme analysis can be effectively simplified using the RO model. Nevertheless, very little research about the RO model and stability analysis of Vienna rectifier has been reported. To deal with those aforementioned issues, using the two-port network theory, the corresponding impedance transfer functions based on the RO small-signal model in the $d$ - $q$ domain must be derived.

\section{Reduced order averaged model of Vienna rectifier}

Without loss of generality, the circuit topology of the three-phase three-level boost-type Vienna rectifier [23] which is adopted in this paper is shown as Fig. 3. It consists of six diodes and three bidirectional switching units $\left(Q_{\mathrm{a}}, Q_{\mathrm{b}}, Q_{\mathrm{c}}\right)$.

To simplify the analysis, the following assumptions are made.

1) The switching frequency is much higher than the grid frequency and the output voltage and grid current are all in steady state.

2) The DC-link capacitor is sufficiently large that, as a result, the output DC voltage is constant during every switching period, and the neutral voltage is zero.

In terms of the AC current signs and the switching states, according to the Kirchhoff equations of the elementary circuits, the circuit equation of the Vienna rectifier is written as:

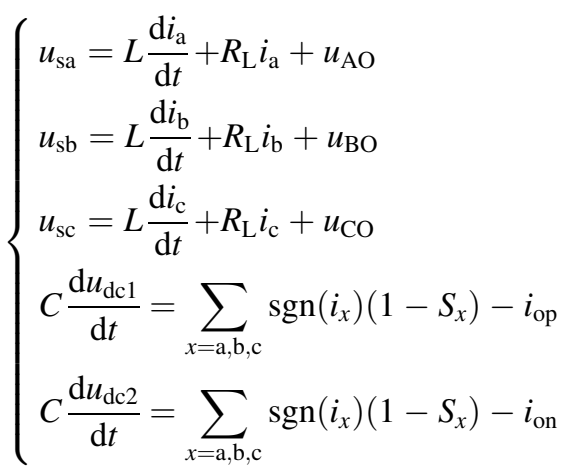

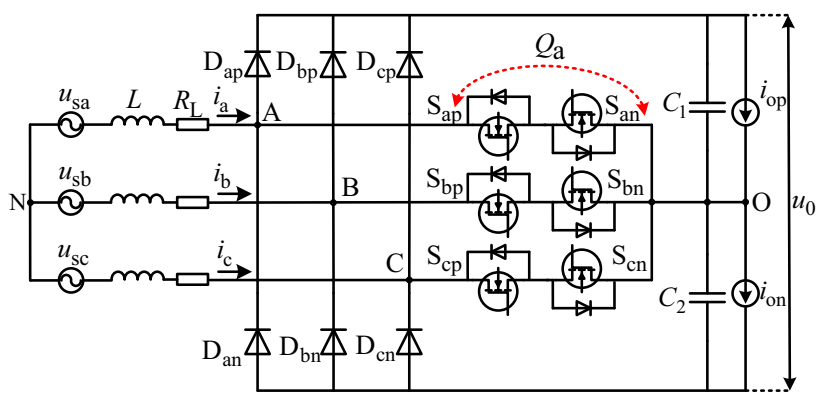

Fig. 3 Topology of three-phase Vienna rectifier 
$S_{x}= \begin{cases}0 & Q_{x} \text { is off } \\ 1 & Q_{x} \text { is on }\end{cases}$

$\operatorname{sgn}\left(i_{x}\right)=\left\{\begin{array}{cl}-1 & i_{x}<0 \\ 1 & i_{x}>0\end{array}\right.$

where $S_{x}$ is the switching function of switch $Q_{x} ; \operatorname{sgn}\left(i_{x}\right)$ is the threshold function.

Since the Vienna rectifier is a current force converter, the pole voltage $\left(u_{\mathrm{AO}}, u_{\mathrm{BO}}, u_{\mathrm{CO}}\right)$ is determined by the switching state and the polarity of the AC phase current [24].

The expression of $u_{\mathrm{AO}}$ is shown as:

$\left\{\begin{array}{l}u_{\mathrm{AO}}=\frac{u_{0}}{2} \operatorname{sgn}\left(i_{\mathrm{a}}\right)\left(1-S_{\mathrm{a}}\right) \\ u_{\mathrm{BO}}=\frac{u_{0}}{2} \operatorname{sgn}\left(i_{\mathrm{b}}\right)\left(1-S_{\mathrm{b}}\right) \\ u_{\mathrm{CO}}=\frac{u_{0}}{2} \operatorname{sgn}\left(i_{\mathrm{c}}\right)\left(1-S_{\mathrm{c}}\right)\end{array}\right.$

Assuming that the average switching time of switch $Q_{x}$ is $T_{x}$ and the switching period is $T_{\mathrm{s}}$, the duty ratio of switch $Q_{x}$ is defined as:

$\left\{\begin{array}{l}d_{\mathrm{a}}=\operatorname{sgn}\left(i_{\mathrm{a}}\right)\left(1-T_{\mathrm{a}} / T_{\mathrm{s}}\right) \\ d_{\mathrm{b}}=\operatorname{sgn}\left(i_{\mathrm{b}}\right)\left(1-T_{\mathrm{b}} / T_{\mathrm{s}}\right) \\ d_{\mathrm{c}}=\operatorname{sgn}\left(i_{\mathrm{c}}\right)\left(1-T_{\mathrm{c}} / T_{\mathrm{s}}\right)\end{array}\right.$

Assuming that the Vienna rectifier is fully decoupled from the unit power factor control, the following nonlinear $d-q$ equations of the system are obtained as:

$\left\{\begin{array}{l}L \frac{\mathrm{d} i_{d}}{\mathrm{~d} t}=u_{d}-\omega L i_{q}-R_{L} i_{d}+\frac{u_{0}}{2} d_{d} \\ L \frac{\mathrm{d} i_{q}}{\mathrm{~d} t}=u_{q}+\omega L i_{d}+R_{L} i_{q}+\frac{u_{0}}{2} d_{q} \\ C \frac{\mathrm{d} u_{0}}{\mathrm{~d} t}=\frac{3}{2}\left(d_{d} i_{d}+d_{q} i_{q}\right)-i_{\mathrm{op}}-i_{\mathrm{on}}\end{array}\right.$

$\left[\begin{array}{lll}u_{d} & u_{q} & u_{0}\end{array}\right]^{\mathrm{T}}=\boldsymbol{K}\left[\begin{array}{lll}u_{\mathrm{sa}} & u_{\mathrm{sb}} & u_{\mathrm{sc}}\end{array}\right]^{\mathrm{T}}$

$\left[\begin{array}{lll}i_{d} & i_{q} & i_{0}\end{array}\right]^{\mathrm{T}}=\boldsymbol{K}\left[\begin{array}{lll}i_{\mathrm{sa}} & i_{\mathrm{sb}} & i_{\mathrm{sc}}\end{array}\right]^{\mathrm{T}}$

$\left[\begin{array}{lll}d_{d} & d_{q} & d_{0}\end{array}\right]^{\mathrm{T}}=\boldsymbol{K}\left[\begin{array}{lll}d_{\mathrm{a}} & a d_{\mathrm{b}} & d_{\mathrm{c}}\end{array}\right]^{\mathrm{T}}$

where $\boldsymbol{K}$ is the abc/dq0 transform matrix.

Assuming that the Vienna rectifier operates under a unity-power factor condition, the nonlinear averaged model of (12) is firstly linearized around the nominal static operating point. The nominal static operating point is defined as:

$\left\{\begin{array}{l}U_{d}=\sqrt{2} U_{\mathrm{s}} \\ I_{d}=\sqrt{2} I_{\mathrm{s}}=2 i_{0} / D_{d} \\ D_{q}=-2 \omega L I_{d} / u_{0}=-2 \sqrt{2} \omega L I_{\mathrm{s}} / u_{0} \\ D_{d}=u_{d} / u_{0}=2 \sqrt{2} U_{\mathrm{s}} / u_{0} \\ i_{q}^{*}=0\end{array}\right.$ where $U_{\mathrm{s}}$ is the root mean square (RMS) value of the source line-to-neutral voltage; $I_{\mathrm{s}}$ is the RMS value of the line current; $i_{0}$ is the output current of the system $\left(i_{0}=i_{\text {op }}+i_{\text {on }}\right)$.

Linearizing the average model around its steady-state operating point results in a small-signal model, and ignoring the higher order factors, the following linear state model can be derived as:

$$
\begin{aligned}
{\left[\begin{array}{c}
\frac{\mathrm{d} \hat{i}_{d}}{\mathrm{~d} t} \\
\frac{\mathrm{d} \hat{i}_{q}}{\mathrm{~d} t} \\
\frac{\mathrm{d} \hat{u}_{0}}{\mathrm{~d} t}
\end{array}\right]=} & {\left[\begin{array}{ccc}
\frac{R}{2 L} & \omega & -\frac{U_{\mathrm{s}} \sqrt{2}}{L u_{0}} \\
-\omega & \frac{R}{2 L} & \frac{\omega I_{\mathrm{s}} \sqrt{2}}{u_{0}} \\
\frac{3 \sqrt{2} U_{\mathrm{s}}}{C u_{0}} & -\frac{3 \sqrt{2} L \omega U_{\mathrm{s}}}{C u_{0}} & 0
\end{array}\right]\left[\begin{array}{c}
\hat{i}_{d} \\
\hat{i}_{q} \\
\hat{u}_{0}
\end{array}\right] } \\
+ & {\left[\begin{array}{ccccc}
\frac{1}{L} & 0 & 0 & -\frac{u_{0}}{2 L} & 0 \\
0 & \frac{1}{L} & 0 & 0 & -\frac{u_{0}}{2 L} \\
0 & 0 & -\frac{1}{C} & \frac{3 \sqrt{2} I_{\mathrm{s}}}{2 C} & 0
\end{array}\right]\left[\begin{array}{c}
\hat{u}_{d} \\
\hat{u}_{q} \\
\hat{i}_{0} \\
\hat{d}_{d} \\
\hat{d}_{q}
\end{array}\right]=\boldsymbol{A x}+\boldsymbol{B u} }
\end{aligned}
$$

Here, the superscript ' $\wedge$ ' represents the disturbance of corresponding variable. The small signal linear models with small signal disturbance are described in (16). Considering the SISO linear regulators [22], the control scheme and small signal stability analysis can be effectively simplified. For this purpose, the reduced small-signal representation of the Vienna rectifier would be derived. Then, the linearized small signal model of the Vienna rectifier of the $d-d$ channel in the $d-q$ domain is written as:

$$
\begin{aligned}
{\left[\begin{array}{l}
\frac{\mathrm{d} \hat{i}_{d}}{\mathrm{~d} t} \\
\frac{\mathrm{d} \hat{u}_{0}}{\mathrm{~d} t}
\end{array}\right]=} & {\left[\begin{array}{cc}
\frac{R}{2 L} & -\frac{D_{d}}{2 L} \\
\frac{3 D_{d}}{2 C} & 0
\end{array}\right]\left[\begin{array}{l}
\hat{i}_{d} \\
\hat{u}_{0}
\end{array}\right] } \\
& +\left[\begin{array}{ccc}
\frac{1}{L} & 0 & -\frac{u_{0}}{2 L} \\
0 & -\frac{1}{C} & \frac{3 I_{d}}{2 C}
\end{array}\right]\left[\begin{array}{l}
\hat{u}_{d} \\
\hat{i}_{0} \\
\hat{d}_{d}
\end{array}\right]
\end{aligned}
$$

The control output equation is derived as:

$$
\begin{aligned}
& {\left[\begin{array}{c}
\hat{i}_{d} \\
\hat{u}_{0}
\end{array}\right]=\left[\begin{array}{ll}
1 & 0 \\
0 & 1
\end{array}\right]\left[\begin{array}{c}
\hat{i}_{d} \\
\hat{u}_{0}
\end{array}\right]+\left[\begin{array}{lll}
0 & 0 & 0 \\
0 & 0 & 0
\end{array}\right]\left[\begin{array}{c}
\hat{u}_{d} \\
\hat{i}_{0} \\
\hat{d}_{d}
\end{array}\right]=\boldsymbol{C} \boldsymbol{x}_{1}+\boldsymbol{D} \boldsymbol{u}_{1}} \\
& \boldsymbol{Y}(s)=\left[\boldsymbol{C}(s \boldsymbol{I}-\boldsymbol{A})^{-1} \boldsymbol{B}+\boldsymbol{D}\right] \boldsymbol{U}(s)
\end{aligned}
$$


where $\boldsymbol{Y}(s)$ denotes output vector; $\boldsymbol{U}(s)$ denotes the control input vector.

The frequency-domain representation of the Vienna rectifier is obtained by applying the Laplace transform of (19). It is shown as:

$$
\left[\begin{array}{c}
\hat{i}_{d} \\
\hat{u}_{0}
\end{array}\right]=\left[\begin{array}{lll}
G_{11}(s) & G_{12}(s) & G_{13}(s) \\
G_{21}(s) & G_{22}(s) & G_{23}(s)
\end{array}\right]\left[\begin{array}{c}
\hat{u}_{d} \\
\hat{i}_{0} \\
\hat{d}_{d}
\end{array}\right]
$$

Based on (16)-(20), the full list of the transfer functions is given as follows:

$$
\begin{aligned}
& G_{11}(s)=\frac{\hat{i}_{d}}{\hat{u}_{d}}=\frac{s}{L} \frac{1}{\operatorname{den}(s)} \\
& G_{12}(s)=\frac{\hat{i}_{d}}{\hat{i}_{0}}=\frac{D_{d}}{2 L C} \frac{1}{\operatorname{den}(s)} \\
& G_{13}(s)=\frac{\hat{i}_{d}}{\hat{d}_{d}}=-\frac{1}{2 L}\left(u_{0} s+\frac{3 i_{0}}{C}\right) \frac{1}{\operatorname{den}(s)} \\
& G_{21}(s)=\frac{\hat{u}_{0}}{\hat{u}_{d}}=\frac{3 D_{d}}{2 L C} \frac{1}{\operatorname{den}(s)} \\
& G_{22}(s)=\frac{\hat{u}_{0}}{\hat{i}_{0}}=-\frac{1}{C}\left(s+\frac{R_{\mathrm{L}}}{L}\right) \frac{1}{\operatorname{den}(s)} \\
& G_{23}(s)=\frac{\hat{u}_{0}}{\hat{d}_{d}}=\frac{3 i_{0}}{D_{d} C}\left(s+\frac{R_{\mathrm{L}}}{L}-\frac{D_{d}^{2} u_{0}}{4 \operatorname{Li} i_{0}}\right) \frac{1}{\operatorname{den}(s)} \\
& \operatorname{den}(s)=s^{2}+\frac{R_{\mathrm{L}}}{L} s+\frac{3 D_{d}^{2}}{4 L C}
\end{aligned}
$$

\section{Impendence analysis of Vienna rectifier in synchronous $d-q$ frame}

In this part, the corresponding impedance transfer functions are computed based on the schemer model. The control diagram of the Vienna rectifier based on the carried-SVM in the $d-q$ domain is shown in Fig. 4 , where SVPWM stands for space vector pulse width modulation. It is compatible with any $d-q$ frame alignment of the input $\mathrm{AC}$ voltages. With this control scheme, the unity power factor is achieved by the proportional-integral (PI) controller and the internal phase-locked loop (PLL). In addition, the PLL aligns the converter with the existent $d$ $q$ domain. The DC-link voltage $u_{0}$ must be assumed as constant, because the rectifier is regulated as an AFE. Compared with the previous modeling method, the proposed method has several special features. It ensures $d$ $q$ frame compatibility. On the other hand, it achieves all control dynamics.

To simplify the analysis, the equivalent carrier-based PWM modulation method is adopted. The corresponding

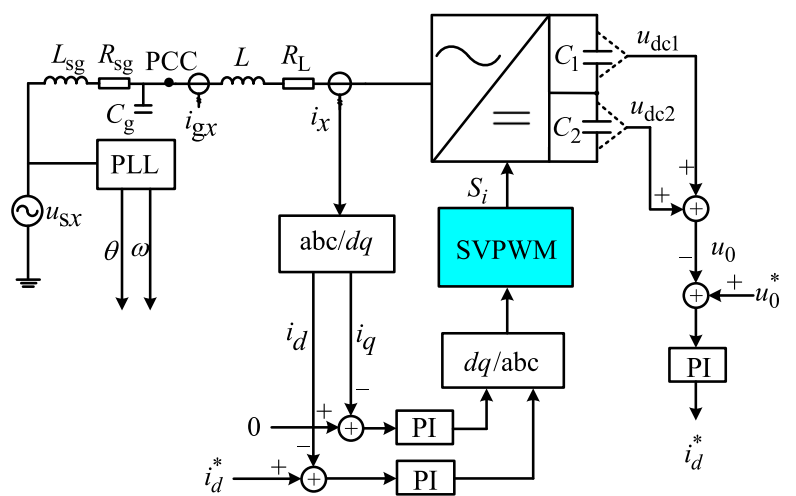

(a) Control block diagram
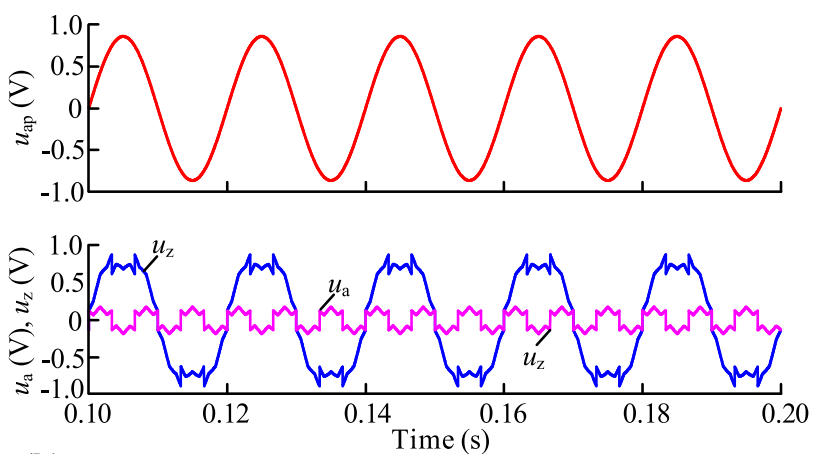

(b) Waveform before and afterinjection of zero-sequence component

Fig. 4 Control block diagram of Vienna rectifier in $d-q$ domain

modulation waveforms without and within zero-sequence injection are shown in Fig. 4. The modulation index $\left(M_{\mathrm{r}}\right)$ is set to $\sqrt{3} / 2, u_{\text {ap }}$ is the waveform before the injection of common-mode component, $u_{\mathrm{z}}$ is the common-mode component, $u_{\mathrm{a}}$ is the waveform after the injection of the common-mode component.

Assuming the three-phase Vienna rectifier system is fully decoupled, then, the dynamic characteristics of the Vienna rectifier are determined by the $d-d$ channel. The control block diagram of the Vienna rectifier based on the RO model is shown in Fig. 5. Here, $G_{\mathrm{PIu}}(s)$ and $G_{\mathrm{PIi}}(s)$ are the PI controller transfer functions of outer voltage loop and inner current loop, respectively. $G_{\mathrm{SVM}}(s)$ is the transfer function of the carried-SVM, which consisted of the modulation and the delay link. The modulation transfer function of carried-SVM [25, 26] is obtained as:

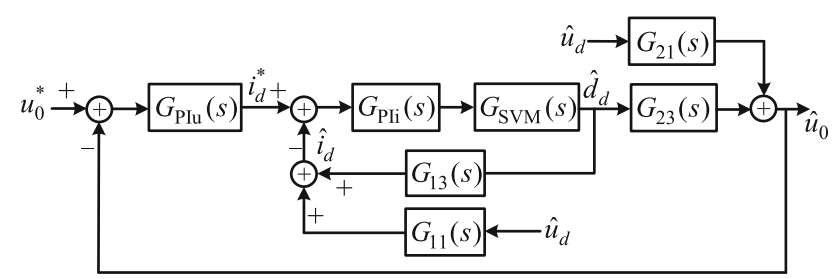

Fig. 5 Control block diagram of Vienna rectifier in $d$ - $d$ channel 
$G_{\mathrm{SVM}, \mathrm{r}}=\frac{1}{M_{\mathrm{r}}}$

When the modulation model is processed by the digital signal, a delay time may be introduced by the sampling and the introduction of a zero-order holder. When the sampling period of the signal is half that of the switching cycle, the expression of carried-SVM modulation method with delay link $G_{\text {delay }}(s)$ is obtained as:

$G_{\mathrm{SVM}}(s)=G_{\text {delay }}(s) G_{\mathrm{SVM}, \mathrm{r}}(s) \approx \frac{1}{M_{\mathrm{r}}} \frac{1}{1.5 T_{\mathrm{S}}+1}$

The current loop transfer function of RO small signal control according to Fig. 5 is shown as:

$G_{\mathrm{c}}(s)=G_{\mathrm{PIi}}(s) G_{\mathrm{SVM}}(s) G_{13}(s)$

At the same time, the transfer function of the voltage loop is written as:

$G_{\mathrm{ud}}(s)=\frac{G_{\mathrm{PIu}}(s) G_{\mathrm{PIi}}(s) G_{23}(s) G_{\mathrm{SVM}}(s)}{1+G_{\mathrm{PIi}}(s) G_{13}(s) G_{\mathrm{SVM}}(s)}$

Based on the above analysis, the close-loop transfer function of linear two ports based on the RO model yields as:

$$
\left[\begin{array}{c}
\hat{i}_{d} \\
\hat{u}_{0}
\end{array}\right]=\left[\begin{array}{ccc}
G_{11 \mathrm{c}}(s) & G_{12 \mathrm{c}}(s) & G_{13 \mathrm{c}}(s) \\
G_{21 \mathrm{c}}(s) & G_{22 \mathrm{c}}(s) & G_{23 \mathrm{c}}(s)
\end{array}\right]\left[\begin{array}{c}
\hat{u}_{d} \\
\hat{i}_{0} \\
\hat{d}_{d}
\end{array}\right]
$$

where the subscript $\mathrm{c}$ on a variable denotes the close-loop transfer function.

It is clear that the expression of open-loop output impedance $Z_{\mathrm{od}, \mathrm{o}}(s)$ is:

$Z_{\text {od,o }}(s)=-G_{22}(s)$

The open-loop input impedance $Z_{\mathrm{id}, \mathrm{o}}(s)$ is:

$Z_{\mathrm{id}, \mathrm{o}}(s)=\frac{1}{G_{11}(s)}$

Using the two-port network theory, the small signal interference caused by $\hat{i}_{d}$ and $\hat{u}_{d}$ is ignored. Considering the transmission relation between $\hat{i}_{0}$ and $\hat{u}_{0}$, according to Fig. 5, the closed-loop output impedance of the Vienna rectifier is derived as:

$$
\begin{aligned}
Z_{\mathrm{od}, \mathrm{c}}(s) & =-G_{22 \mathrm{c}}(s)=\frac{\hat{u}_{0}}{\hat{i}_{0}}=\frac{-G_{22}(s)+G_{12}(s) G_{23}(s) G_{\mathrm{c}}(s)}{1+G_{\mathrm{PIu}}(s) G_{\mathrm{c}}(s) G_{23}(s)} \\
& \approx \frac{-G_{22}(s)}{1+G_{\mathrm{PIu}}(s) G_{\mathrm{c}}(s) G_{23}(s)}
\end{aligned}
$$

The closed-loop input admittance of Vienna rectifier is:

$$
\begin{aligned}
Y_{\mathrm{id}, \mathrm{c}}(s)=G_{11 \mathrm{c}}(s)= & \frac{\hat{i}_{d}}{\hat{u}_{d}}=\frac{G_{11}(s)}{1+G_{\mathrm{c}}(s)}+\frac{G_{11}(s) G_{\mathrm{c}}(s) G_{\mathrm{ud}}(s)}{\left(1+G_{\mathrm{c}}(s)\right)\left(1+G_{\mathrm{ud}}(s)\right)} \\
& -\frac{G_{21}(s) G_{13}(s) G_{\mathrm{PIu}}(s)}{\left(1+G_{\mathrm{c}}(s)\right)\left(1+G_{\mathrm{ud}}(s)\right)} \approx \frac{G_{11}(s)}{1+G_{\mathrm{c}}(s)}
\end{aligned}
$$

The close-loop small signal model of the Vienna rectifier is shown below:

$$
\begin{aligned}
G_{12 \mathrm{c}}(s)= & \frac{\hat{i}_{d}}{\hat{i}_{0}}=\frac{G_{12}(s)}{1+G_{\mathrm{c}}(s)}+\frac{G_{12}(s) G_{\mathrm{c}}(s) G_{\mathrm{ud}}(s)}{\left(1+G_{\mathrm{c}}(s)\right)\left(1+G_{\mathrm{ud}}(\mathrm{s})\right)} \\
& -\frac{G_{22}(s) G_{23}(s) G_{\mathrm{PIu}}(s)}{\left(1+G_{\mathrm{c}}(s)\right)\left(1+G_{\mathrm{ud}}(\mathrm{s})\right)} \\
G_{13 \mathrm{c}}(s)= & \frac{\hat{i}_{d}}{\hat{d}_{d}}=\frac{G_{13}(s) G_{\mathrm{PIi}}(s)}{1+G_{\mathrm{c}}(s)}-\frac{G_{13}(s) G_{\mathrm{PIu}}(s)}{\left(1+G_{\mathrm{c}}(s)\right)\left(1+G_{\mathrm{ud}}(s)\right)} \\
G_{21 \mathrm{c}}(s)= & \frac{\hat{u}_{0}}{\hat{u}_{\mathrm{d}}}=\frac{G_{21}(s)-G_{23}(s) G_{11}(s)}{\left(1+G_{\mathrm{c}}(s)\right)\left(1+G_{\mathrm{ud}}(s)\right)} \\
G_{23 \mathrm{c}}(s)= & \frac{\hat{u}_{0}}{\hat{d}_{\mathrm{d}}}=\frac{G_{23}(s) G_{\mathrm{PIu}}(s) G_{\mathrm{c}}(s)}{1+G_{\mathrm{ud}}(s)}
\end{aligned}
$$

\section{Small-signal stability analysis and virtual impedance design}

In order to validate the theoretical analysis proposed, a simulation model and experimental prototype of the Vienna rectifier are built. The test parameters are kept consistent with the theoretical analysis. Unless otherwise noted, in all the simulation cases the grid phase voltage is $380 \mathrm{~V} / 50 \mathrm{~Hz}$, the grid voltage frequency is $50 \mathrm{~Hz}$, the DC bus voltage is $750 \mathrm{~V}$, the DC bus capacitor is $1080 \mu \mathrm{F}$, and the switching frequency adopted in this paper is $50 \mathrm{kHz}$. The parameters of grid LC filter I is as below, $L_{\mathrm{sg}}=0.3$ $\mathrm{mH}, R_{\mathrm{sg}}=0.02 \Omega, C_{\mathrm{g}}=20 \mu \mathrm{F}, R_{\mathrm{cg}}=0.03 \Omega$. For LC filter I, the value of $C_{\mathrm{g}}$ is $5 \mu \mathrm{F}$. The parameters of PI regulator involved is as list, the $K_{\mathrm{p}}$ of outer loop is 0.1 and $K_{\mathrm{i}}$ is 10 ,the $K_{\mathrm{p}}$ of inner loop is 0.01 and $K_{\mathrm{i}}$ is 20 .

\subsection{Small signal stability analysis of Vienna rectifier with LC filter}

To validate the correctness of the proposed method, the small signal stability between the Vienna rectifier and the grid impedance is analyzed. As shown in Fig. 1, the output impedance of the source subsystem in the $d-q$ domain is derived as: 


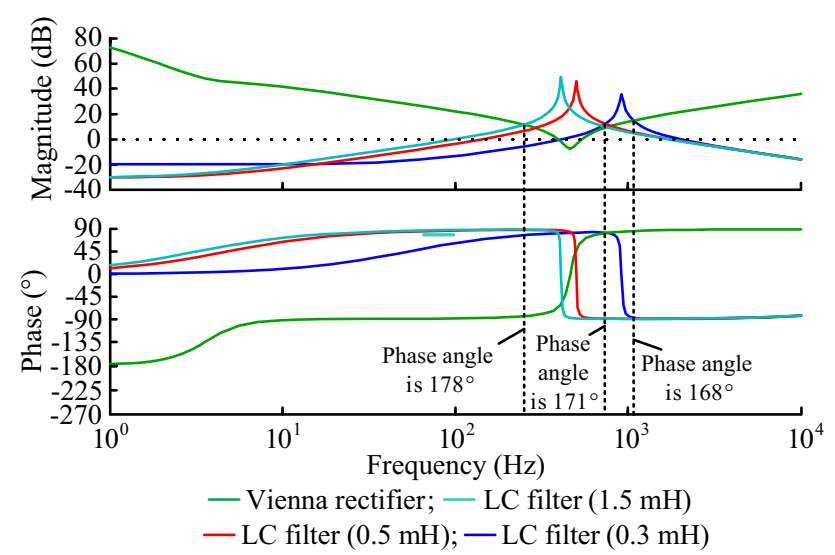

(a) Variable inductance

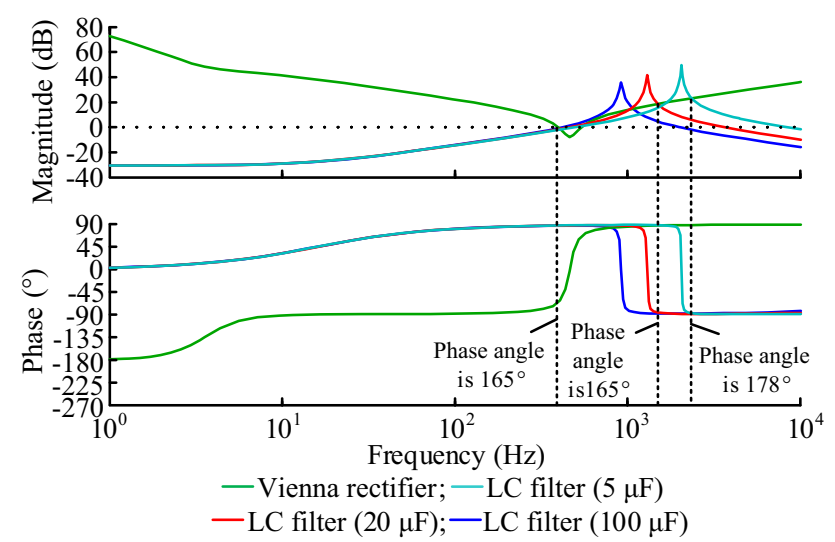

(b) Variable capacitance

Fig. 6 Impedance of Vienna rectifier and LC filter

$$
Z_{\mathrm{sg}, d d}(s)=Z_{\mathrm{sg}, q q}(s)=\frac{\left(L_{\mathrm{sg}} s+R_{\mathrm{sg}}\right)\left(R_{\mathrm{cg}} C_{\mathrm{g}} s+1\right)}{L_{\mathrm{sg}} C_{\mathrm{g}} s^{2}+\left(R_{\mathrm{sg}}+R_{\mathrm{cg}}\right) C_{\mathrm{g}} s+1}
$$

The impedance of the Vienna rectifier and source subsystem (i.e. the LC filter) is shown in Fig. 6a. It shows that the amplitude of the grid impedance is greatly increased at the resonance frequency. The cross section is shown near the resonance frequency of the output impedance. In order to simplify the computation, the disturbance term was ignored. It can be seen that the cascade system of the Vienna rectifier and LC filter will be unstable with the decrease of filter capacitor $C_{\mathrm{g}}$. The impedance ratio curve of phase difference is nearly $180^{\circ}$ as the filter capacitor decreases to $20 \mu \mathrm{F}$. However, it should be pointed out that there must be a certain absolute error because the disturbance term of (36) was ignored. Considering many simulation and experiment results, the derived error of phase between the theoretical calculation and actual system is about $5^{\circ}$. In order to maintain the system in a stable condition, the phase difference between the impedance of LC filter and Vienna rectifier should be kept smaller than $175^{\circ}$.

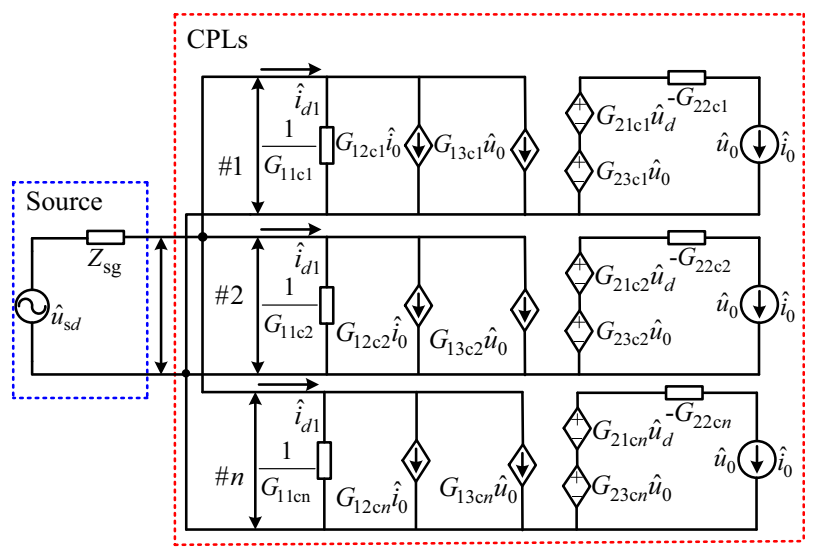

Fig. 7 Reduced order linear two-port model of Vienna rectifier in parallel system

\subsection{Stability analysis of system with increasing number of CPLs}

In an electrical system, it is difficult for a single power module to meet the demand of a high-power power supply system. However, with an increasing number of CPLs, oscillations and over voltages maybe induced. We analyse the stability of the system with an increased number of Vienna rectifiers in parallel operation. The reduced order linear two-port model of a multi Vienna rectifier parallel operation system is shown in Fig. 7. It should be pointed out that the influence of cross-coupling is ignored, and all the modules work in the same stable state.

The equivalent input impedance of a multi Vienna rectifier parallel operation system can be expressed as:

$Z_{\mathrm{eq}}(s)=\frac{1}{G_{11 \mathrm{c} 1}(s)+G_{11 \mathrm{c} 2}(s)+\cdots+G_{11 \mathrm{c} n}(s)}=\frac{G_{11 \mathrm{c} 1}(s)}{n}$

where $G_{11 \mathrm{c} 1}(\mathrm{~s}), G_{11 \mathrm{c} 2}(\mathrm{~s}), \ldots, G_{11 \mathrm{c} n}(\mathrm{~s})$ are the input impedances of the Vienna rectifiers; $Z_{\mathrm{eq}}(s)$ is the equivalent input impedance; $n$ is the number of multi Vienna rectifiers in parallel operation.

The amplification Nyquist curve of the system with the increase in number of Vienna rectifier is shown in Fig. 8. It

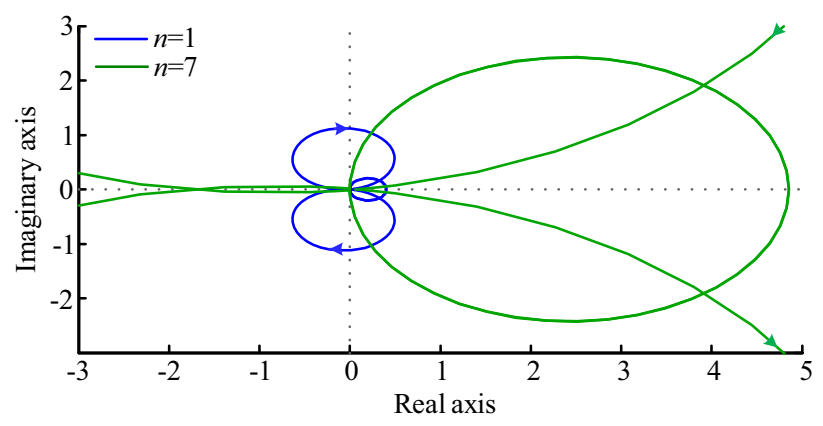

Fig. 8 Amplification Nyquist curve with change number of rectifiers 
is clear that the Nyquist curve gradually becomes close to the point $(1,0)$. However, when the number of parallel modules is increased to 7 , the parallel system becomes unstable in the case of the given power grid distribution parameters.

\subsection{Design method of virtual impedance}

Based on the previous discussion, for unstable cascade AC systems, the phase difference between the input impedance of a Vienna rectifier and an LC filter should be kept smaller than $180^{\circ}$ [27]. To prevent unstable performance, a series damping resistance can be introduced to the filter capacitor branch. However, the power loss of the system is then increased. In addition, it suppresses attenuation ability at high frequency [27, 28]. Based on the proposed criterion, an impedance regulator is then proposed, in which the voltages of the filter capacitors are applied to the modulation waves through an appropriate regulator. Taking the $d-d$ channel of the Vienna rectifier as an example, the proposed method is shown in Fig. 9.

As shown in the Fig. 9, it is clear that the transfer function between the duty ratio and the grid voltage is changed with the proposed impedance regulator, and the equation of $\hat{d}_{d}$ is changed to:

$\hat{d}_{d}=G_{\mathrm{PIu}}(s) G_{\mathrm{PIi}}(s) G_{\mathrm{SVM}}(s) \hat{u}_{0}+G_{\mathrm{PIi}} G_{\mathrm{SVM}} \hat{i}_{d}+k G_{\mathrm{SVM}}(s) \hat{u}_{d}$

where $k$ is the virtual impedance regulator.

Substituting (43) into (17), the input impedance of the Vienna rectifier with the proposed method is rearranged to:

$Z_{\mathrm{id}, \mathrm{i}, \mathrm{c}}(s)=-\frac{Z_{\mathrm{id}, \mathrm{c}}(s)}{k G_{\mathrm{SVM}}(s) u_{0} / 2}$

To maintain the stability of cascade AC systems, the phase angle in the cross section $\left[f_{1}, f_{2}\right]$ must be kept between $\left[-90^{\circ}, 90^{\circ}\right]$. This means that the input impedance of CPL must be in the quadrant I or IV in the complex plane. The input impedance in the complex plane is shown in Fig. 10.

Based on Fig. 9, the input impedance of the Vienna rectifier with virtual impedance will be rearranged as:

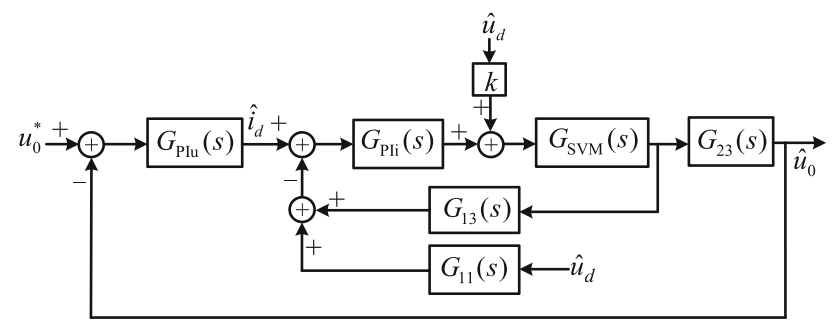

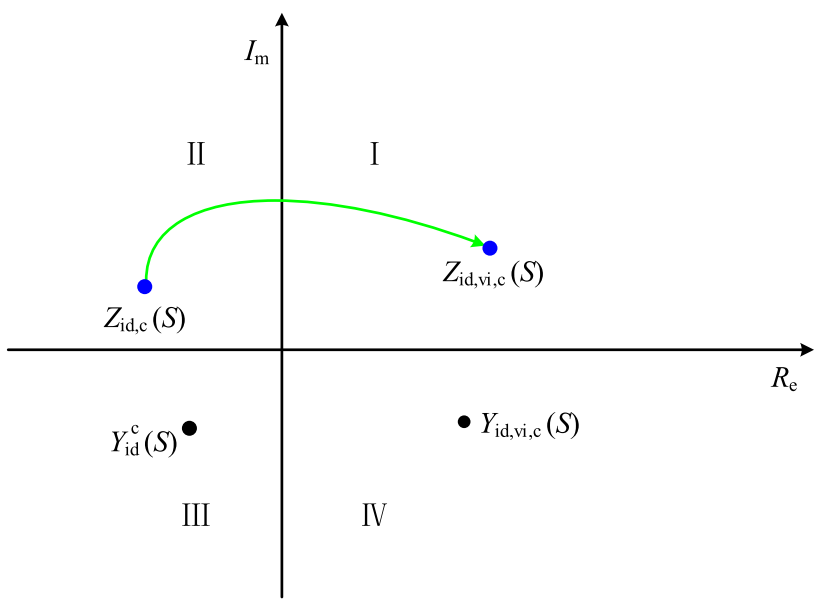

Fig. 10 Input impedance in complex plane

$Z_{\mathrm{id}, \mathrm{vi}, \mathrm{c}}(s)=\frac{Z_{\mathrm{id}, \mathrm{c}}(s)}{1-k G_{\mathrm{SVM}}(s) u_{0} / 2}$

The input impedance with the impedance regulator introduced is adjusted from the left half plane of the complex plane to the right half plane [27]. The real part $\left(R_{\mathrm{e}}\right)$ of input impedance $Z_{\text {id,vi,c }}(s)$ with the impedance regulator introduced in the cross section $\left[f_{1}, f_{2}\right]$ should satisfy:

$R_{\mathrm{e}}\left(Z_{\text {id, vi,c }}(s)\right) \geq 0$

The coefficient of impedance regulation $k$ can be calculated by (46).

\section{Digital simulation and experiment verification}

\subsection{Simulation results}

To verify the proposed stability criterion and impedance regulator design method, a whole digital simulation model based on the PSIM is built. The value of the filter inductance is set at $0.35 \mathrm{mH}$. Note that in all simulation cases, the simulation and experimental parameters are consistent with the theoretical analysis.

Figure 11 shows the simulation results of grid current with a change of LC filter. The grid current under ideal grid power is shown in Fig. 11a. As Fig. 11b shows, the grid current and voltage at the PCC might be distorted when the distributed capacitance is about $20 \mu \mathrm{F}$. If the capacitance decreased to $5 \mu \mathrm{F}$, the grid current will become unstable (Fig. 11c). Obviously, the performance is greatly improved with the introduction of an impedance regulator as shown in Fig. 11d.

Fig. 9 Block diagram with virtual impedance 


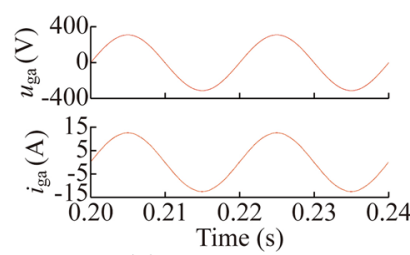

(a) Ideal grid power

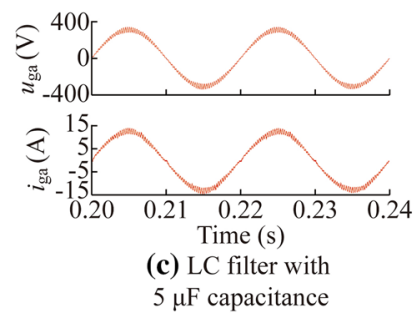

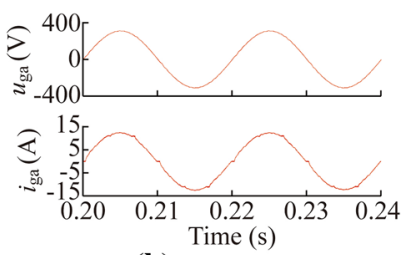

(b) LC filter with

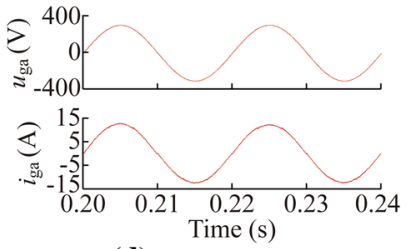

(d) Virtual impedance
$20 \mu \mathrm{F}$ capacitance

Fig. 11 Simulation waveforms under different LC filters

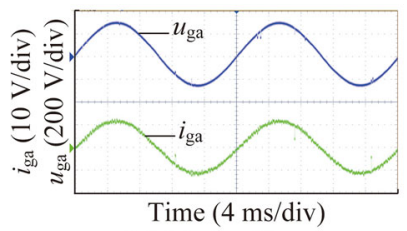

(a) Ideal grid power

(without LC filter)

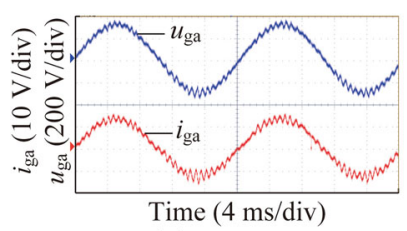

(c) LC filter II

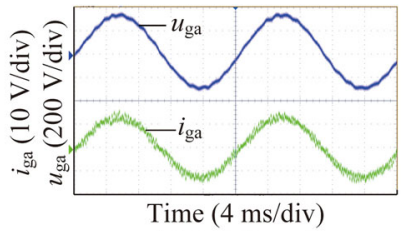

(b) LC filter I

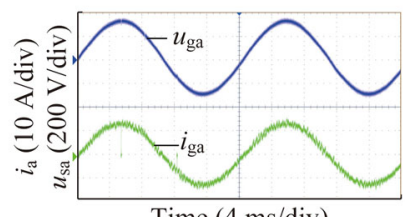

Time (4 ms/div) (d) Virtual impedance

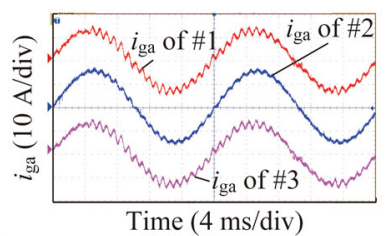

(e) Multi Vienna rectifier parallel operation

Fig. 12 Steady-state experimental waveforms

\subsection{Experimental results}

To verify the above theoretical analysis, an experimental prototype is constructed. The grid current and voltage under ideal grid is shown in Fig. 12a. Figure 12b is the output waveform with a $20 \mu \mathrm{F}$ filter capacitor. Figure $12 \mathrm{c}$ is the output waveform of grid current with a $5 \mu \mathrm{F}$ filter capacitor. Figure $12 \mathrm{~d}$ is the grid current with the proposed impedance regulator. It shows that the quality of grid current is effectively improved with the proposed methods. The experimental results agree very well with the simulation. As a result, the theoretical analysis is validated.
To avoid the influence of unknown disturbance on parallel operation,a model of the parallel system is built based on the Real Time-Laboratory (RT-LAB). The grid current of a \#1 and \#2 Vienna rectifier is shown as Fig. 12. It is clear that the grid current is unstable. All the above results are consistent with the theoretical analysis.

\section{Conclusion}

A simplified small signal stability criterion is presented for the three-phase unity-power factor Vienna cascaded AC systems. Based on this criterion, an input impedance regulator design method to stabilize the unstable cascaded AC systems is proposed. In order to verify the correctness and superiority of the proposed methods, a complete simulation is carried out and an RT-LAB experimental model is built. The results demonstrate that the stability of a cascaded AC system is fully determined by the SISO return-ratio of $d$ $d$ channel impedances, and with the proposed impedance regulator the system stability is greatly improved and is not sensitive to the grid impedance. In addition, the proposed criterion can predict and guarantee the stable operation of a cascaded system during the design process, and the proposed method could also be used for other three-phase topologies.

Acknowledgements This work was supported by the National Natural Science Fund of China (No. 51677151), National Natural Science Youth Fund of China (No. 51507138), the Major Scientific and Technological Innovation Projects of Shaanxi Province, China (No. 2015ZKC02-01) and International Exchange And Cooperation Project of Key R\&D Program in Shaanxi (No. 2017KW-035).

Open Access This article is distributed under the terms of the Creative Commons Attribution 4.0 International License (http:// creativecommons.org/licenses/by/4.0/), which permits unrestricted use, distribution, and reproduction in any medium, provided you give appropriate credit to the original author(s) and the source, provide a link to the Creative Commons license, and indicate if changes were made.

\section{References}

[1] Dang CL, Tong XQ, Huang JJ et al (2017) The neutral pointpotential and current model predictive control method for Vienna rectifier. J Frankl Inst 354(17):7605-7623

[2] Dang CL, Tong XQ, Huang JJ et al (2017) QPR and duty ratio feed-forward control for Vienna rectifier of HVDC supply system. IEEJ Trans Electr Electron Eng 12(4):501-509

[3] Kolar JW, Zach FC (1997) A novel three-phase utility interfaces minimizing line current harmonics of high-power tele communications rectifier modules. IEEE Trans Ind Electron 44(4):456-466 
[4] Ma H, Xie YX, Shi ZY (2016) Improved direct power control for Vienna-type rectifiers based on sliding mode control. IET Power Electron 9(3):427-434

[5] Wen B, Boroyevich D, Mattavelli P et al (2012) Experimental verification of the generalized Nyquist stability criterion for balanced three-phase AC systems in the presence of constant power loads. In: Proceedings of IEEE energy conversion congress and exposition (ECCE), Raleigh, USA, 15-20 September 2012, pp 3926-3933

[6] Liu Z, Liu JJ, Bao WH et al (2015) Infinity-norm of impedancebased stability criterion for three-phase AC distributed power systems with constant power loads. IEEE Trans Power Electron 30(6):3030-3043

[7] Cespedes M, Sun J (2014) Impedance modeling and analysis of grid connected voltage-source converters. IEEE Trans Power Electron 29(3):1254-1261

[8] Middlebrook RD (1976) Input filter consideration in design and application of switching regulators. In: Proceedings of IEEE industry applications society annual meeting, Chicago, USA, 10-11 October 1976, pp 94-107

[9] Rygg A, Molinas M, Zhang C et al (2016) A modified sequencedomain impedance definition and its equivalence to the $d-q$ domain impedance definition for the stability analysis of AC power electronic systems. IEEE J Emerg Sel Top Power Electron 4(4):1383-1396

[10] Vesti S, Suntio T, Oliver JA et al (2013) Impedance based stability and transient-performance assessment applying maximum peak criteria. IEEE Trans Power Electron 28(5):2099-2104

[11] Mao HC, Boroyevich DS, Lee FCY (1998) Novel reduced-order small-signal model of a three-phase PWM rectifier and its application in control design and system analysis. IEEE Trans Power Electron 13(3):511-521

[12] Belkhayat M (1997) Stability criteria for AC power systems with regulated loads. Dissertation, Purdue University

[13] Feng X, Liu J, Lee FC (2002) Impedance specifications for stable DC distributed power systems. IEEE Trans Power Electron 17(2):157-162

[14] Francis G, Burgos R, Boroyevich D et al (2011) An algorithm and implementation system for measuring impedance in the $D$ $Q$ domain. In: Proceedings of 2011 IEEE energy conversion congress and exposition (ECCE), Phoenix, USA, 17-22 September 2011, pp 3221-3228

[15] Phattanasak M, Ghoachani RG, Martin JP (2016) Lyapunovbased control and observer of a boost converter with LC input filter and stability analysis. In: Proceedings of 2016 international conference on electrical systems for aircraft, railway, ship propulsion and road vehicles \& international transportation electrification conference (ESARS-ITEC), Toulouse, France, 2-4 November 2016, pp 1-6

[16] Cao WC, Ma YW, Liu Y (2017) $D-Q$ impedance based stability analysis and parameter design of three-phase inverter-based AC power systems. IEEE Trans Ind Electron 99(3):1-11

[17] Wen B, Boroyevich D, Burgos R (2015) Small-signal stability analysis of three-phase AC systems in the presence of constant power loads based on measured $d-q$ frame impedances. IEEE Trans Power Electron 30(10):5952-5963

[18] Debranjan M, Debaprasad K (2015) Voltage sensor less control of the three-level three-switch Vienna rectifier with programmable input power factor. IET Power Electron 8(8):1349-1357

[19] Freddy FB, Hugo VB, Luis MS et al (2014) Control of a threephase AC/DC Vienna converter based on the sliding mode lossfree resistor approach. IET Power Electron 7(5):1073-1082

[20] Lin HW, Jia CX, Vasquez JC (2017) Angle stability analysis for voltage-controlled converter. IEEE Trans Ind Electron 99(3): $1-11$

[21] Burgos R, Boroyevich D, Wang F et al (2010) On the AC stability of high power factor three-phase rectifiers. In: Proceedings of 2010 IEEE energy conversion congress and exposition (ECCE), Atlanta, USA, 12-16 September 2010, pp 2047-2054

[22] Youssef NBH, Al-Haddad K, Kanaan HY (2008) Implementation of a new linear control technique based on experimentally validated small-signal model of three-phase three-level boosttype Vienna rectifier. IEEE Trans Ind Electron 55(3):1666-1676

[23] Gabriel HPO, Maswood AI, Lim ZY (2016) Grid connected three-phase multiple-pole multilevel unity power factor rectifier with reduce components count. IET Power Electron 9(7):1437-1444

[24] Thandapani T, Paramasivam S, Karpagam R (2015) Modelling and control of Vienna rectifier a single phase approach. IET Power Electron 8(12):2471-2482

[25] Lee JS, Lee KB (2015) Carrier-based discontinuous PWM method for Vienna rectifiers. IEEE Trans Power Electron 30(6):2896-2900

[26] Hang LJ, Li B, Zhang M et al (2013) Space vector modulation strategy for Vienna rectifier and load unbalanced ability. IET Power Electron 6(7):1399-1405

[27] Yu H, Ruan XB, Wang XH et al (2014) Stability analysis of cascade AC system based on three-phase voltage source PWM rectifier. In: Proceedings of 2014 international power electronics and application conference and exposition, Shanghai, China, 5-8 November 2015, pp 847-852

[28] Xue MY, Zhang Y, Kang Y (2012) Full feedforward of grid voltage for discrete state feedback controlled grid-connected inverter with LCL filter. IEEE Trans Power Electron 27(10):4234-4247

Chaoliang DANG received the B.S. degree in electrical engineering from Xi' an Technology University, China, in 2011 and M.S. degree in control theory and control engineering from Lanzhou University of Technology, China, in 2014, respectively. He is currently working toward the $\mathrm{Ph} . \mathrm{D}$. degree in electrical engineering from $\mathrm{Xi}$ 'an University of Technology. Her research interests include analysis of power quality, electronic motor control, the new control strategy in the application of power electronic drives and control of hybrid power filter.

Xiangqian TONG received the B.S. degree from the Shaanxi Institute of Technology, Hanzhong, China, the M.S. degree from Xi' an University of Technology, Xi'an, China, and the Ph.D. degree in electrical engineering from Xi'an Jiaotong University, Xi'an, China, in 1983, 1989 and 2006, respectively. He joined the Xi' an University of Technology in 1989. Since 2002, he has been a professor and the academic leader of electrical engineering with the Xi'an University of Technology. His research interests include the application of power electronics in power system and control of 
power quality, especially the power filter, static synchronous compensator, and high voltage direct current.

Weizhang SONG received the B.S. and M.S. degrees in information and control engineering and $\mathrm{Ph} . \mathrm{D}$. degree in electrical engineering all from Xi'an University of Technology, Xi'an, China, in 2004, 2007 and 2010, respectively. In 2011, he joined the department of Xi'an University of Technology, where he became an associate professor in 2014. From 2014 to 2015, he was a research fellow (postdoctoral fellow) with the Power Electronics, Machines and Control (PEMC)
Group in the University of Nottingham, UK. His research interests include power electronics and $\mathrm{AC}$ drive system, especially matrix converter and Vienna rectifier.

Jingjing HUANG received the M.S. degree and the $\mathrm{Ph} . \mathrm{D}$. degree in electrical engineering from Xi' an Jiaotong University, Xi' an, China, in 2010 and 2014, respectively. She joined the Xi' an University of Technology in 2014. Her research interests include the application of power electronics in power system, control of power quality, and static synchronous compensator. 